\title{
Cognitive Inhibition Correlates with Exercise-Induced Hypoalgesia After Aerobic Bicycling in Pain-Free Participants
}

This article was published in the following Dove Press journal: Journal of Pain Research

\section{H Gajsar (D) \\ C Titze' \\ K Konietzny' \\ $M M^{M e y e r}{ }^{2}$ \\ HB Vaegter (D) ${ }^{3,4}$ \\ MI Hasenbring ${ }^{1,3}$}

'Mind and Pain in Motion Group, Department of Medical Psychology and Medical Sociology, Ruhr University Bochum, Bochum, Germany; ${ }^{2}$ School of Social Sciences, University of Mannheim, Mannheim, Germany; ${ }^{3}$ Department of Clinical Research, Faculty of Health Sciences, University of Southern Denmark, Odense, Denmark; ${ }^{4}$ Pain Research Group, Pain Center, University Hospital Odense, Odense, Denmark
Correspondence: $\mathrm{H}$ Gajsar Mind and Pain in Motion Group, Department of Medical Psychology and Medical Sociology, Ruhr University Bochum, Universitätsstr, I50, Bochum 44809, Germany

Email Gajsar@medpsych.rub.de
Purpose: Exercise-induced hypoalgesia (EIH) is the short-term reduction of pain sensitivity after a single bout of exercise. Descending pain inhibition has been proposed to at least partly underlie EIH. Cognitive inhibition is the ability to inhibit a pre-potent response and has in turn been associated with descending pain inhibition, as indexed by conditioned pain modulation. Therefore, we hypothesized that cognitive inhibition is associated with higher EIH.

Methods: In this cross-sectional study, 37 pain-free participants (16 male, age $27.75 \pm 9.91$ ) completed a stop-signal task assessing cognitive inhibition ability and a control condition in the first session. In the second session, pre-post-test design EIH was assessed by means of aerobic bicycling (15 min., 75\% $\mathrm{VO}_{2} \mathrm{max}$ ) and isometric knee extension (90 sec, 30\% MVC). EIH was assessed with pressure pain thresholds (PPT) and temporal summation of pain (TSP), each at the hand and at the leg. Correlational analyses quantified the associations between cognitive inhibition and EIH change scores.

Results: Better cognitive inhibition correlated with EIH change scores in PPTs after aerobic bicycling at the hand $(r=-0.35,95 \% \mathrm{CI}:-0.57 ;-0.08, p=0.021)$, but not at the leg (rho $=$ $-0.10,95 \%$ CI: $-0.36 ; 0.18, p=0.277)$. No correlations between cognitive inhibition and change in PPTs after isometric knee extension at the hand (rho $=-0.03,95 \% \mathrm{CI}:-0.30 ; 0.25$, $p=0.857$ ) nor at the leg (rho $=-0.03,95 \% \mathrm{CI}:-0.25 ; 0.30, p=0.857)$ were observed. There were no EIH effects after isometric exercise and, generally, no effects of exercise on TSP. Conclusion: This study provides preliminary evidence for the notion that cognitive inhibition might play a supportive role in EIH. Although these results are clearly in need of replication, they accord well with previously reported associations between cognitive inhibition, experimental pain and descending pain inhibition.

Keywords: descending pain inhibition, exercise-induced hypoalgesia, response inhibition, stop-signal task, pressure pain threshold, temporal summation of pain

\section{Introduction}

The existence of endogenous processes capable of pain inhibition is well accepted, and most prominently known from the "pain-inhibits-pain" or conditioned pain modulation phenomenon (CPM). ${ }^{1,2}$ Increasing evidence suggests that pain inhibition can also be triggered by physical exercise: in healthy individuals, even a single bout of exercise leads to a short-termed decrease in pain sensitivity, which is denoted as exercise-induced hypoalgesia $(\mathrm{EIH}) .{ }^{3,4} \mathrm{EIH}$ research suggests that considerable variance in EIH effects exists in patients with chronic pain, and, to a lesser 
extent, in pain-free, healthy participants. ${ }^{3-5}$ Therefore, understanding influential individual differences in painfree participants that might translate to chronic pain can potentially aid in individually tailoring exercise-based pain management interventions. ${ }^{4}$ There is preliminary evidence that EIH declines with increasing age ${ }^{6}$ and is more pronounced in women. ${ }^{7}$ Furthermore, a few studies have addressed self-reported psychological variables, such as pain catastrophizing and kinesiophobia. ${ }^{6,8}$ However, these have yielded either null-findings or inconclusive evidence. $^{6,9-11}$

Concurrently, recent research indicates that cognitive function might affect pain, and in particular, pain inhibition. ${ }^{12,13}$ As both pain inhibition and cognitive function seem to be diminished in chronic pain and with increasing age, the link between the two has gained some attention in recent years. ${ }^{12-19}$ Therefore, another individual difference that might influence EIH worth investigating might be at the level of cognitive function: Here, inhibition is a hallmark of higher-order cognitive functions, which denotes the ability to deliberately suppress dominant, automatic or pre-potent responses. ${ }^{20}$ The ability for cognitive inhibition depends on the neurophysiological integrity of the brain, especially in the prefrontal cortex (PFC) and can be severely impaired after neurological lesions of the brain. ${ }^{21}$ Milder impairments in cognitive inhibition have been linked to personality traits like impulsivity, psychiatric conditions, as well as to chronic pain. ${ }^{13,22,23}$ Furthermore, the individual ability for cognitive inhibition varies considerably between individuals. ${ }^{24,25}$ Quantifying an individual's performance in inhibition tasks is a well-accepted means to measure cognitive inhibition; ${ }^{24}$ the stop-signal task, indexing the inhibition of pre-dominant motor responses, is one such measure of cognitive inhibition. ${ }^{26,27}$

Research on the link between cognitive inhibition and experimental pain indicates that indicates individuals with better cognitive inhibition abilities show higher pain tolerance and a higher CPM response. ${ }^{12,19,28,29}$ In this regard, it has been argued that cognitive inhibition is a supporting cognitive function in the regulation of pain, proposing that cognitive inhibition constitutes a functional part of pain inhibition. Therefore, cognitive inhibition might also be relevant in EIH. ${ }^{12}$ This assumption is further supported considering that EIH, CPM and cognitive inhibition have been associated with activity in the same brain areas, ie prefrontal cortex (PFC) and anterior cingulate cortex (ACC) ${ }^{1,30-33}$ Therefore, it was hypothesized that painfree individuals with better cognitive inhibition, as assessed by performance in a cognitive inhibition task, also show a higher magnitude of EIH.

\section{Methods}

\section{Participants}

Participants were recruited at the Ruhr University Bochum via announcements on notices that were dispersed throughout the campus and posted on social media as well as via word of mouth. Exclusion criteria consisted of an age below 18 years, a history of ongoing neurological or psychiatric disease, cardiovascular disease, infectious disease, metabolic diseases or thyroid malfunction, chronic or acute pain, high-frequent alcohol intake, former and acute regular intake of drugs, regular intake of analgesic medication, hormonal contraception, as well as usage of corticosteroids during the month prior to participation. These criteria were screened via a standardized telephone interview before testing. Participants were also asked to refrain from any pain medication and vigorous exercise for $24 \mathrm{~h}$, and caffeine for $4 \mathrm{~h}$ before the experiment. ${ }^{34}$ Eighty participants were recruited following this procedure. As the present study was part of a larger investigation, 40 participants were excluded from analysis in the present study, because they received experimental manipulations that were assumed to affect EIH. As the present work was an exploratory study within a larger investigation, a sample size calculation for the effect investigated here was not conducted in advance. The remaining 40 participants were included in the present study. The study was conducted in accordance with the Declaration of Helsinki and approved by the ethical review board of the Faculty of Psychology at Ruhr University Bochum (application \#242). All subjects provided written informed consent before participating in the study and were compensated with 20 Euros for their time.

\section{Procedure}

The present study was of a correlational design and part of a larger investigation on emotion regulation of exerciseinduced hypoalgesia (EIH) conducted in 2016 (for the full protocol please see dx.doi.org/10.17504/protocols. io.873hzqn). Note that in this study, additional data on blood pressure and salivary cortisol were obtained, which will be reported elsewhere. Participants completed two testing sessions in a within-subjects design, separated by a minimum of one week. Cognitive inhibition was assessed using a stop-signal task, ${ }^{35}$ and EIH was assessed with two 
different types of exercise: an isometric knee extension and an aerobic bicycling exercise.

In session one, participants were familiarized with the pain sensitivity assessment procedures, that is, pressure pain thresholds (PPTs) and temporal summation of pain (TSP). After a 5-min break, pre- and post-assessments of pain sensitivity were administered immediately before and immediately after a 15-min quiet rest period followed, serving as the control condition in the EIH paradigm. Next, participants completed the stop-signal task. Following this, at the end of session one, an assessment of maximum voluntary contraction force (MVC) of knee extension was conducted as a preparation for the isometric knee extension exercise in the second session.

In session two, an isometric knee extension and an aerobic bicycling exercise were performed in a counterbalanced, randomized order. Pre- and post-measurements for each exercise were obtained immediately before and after exercise. Ratings of perceived painfulness and unpleasantness were obtained about 5 minutes after the post-assessment of PPTs and TSP following each exercise. Intervals of $20 \mathrm{~min}$ utes were kept between the two exercises, as it has been suggested that the hypoalgesic effects of exercise do not last longer than 15 minutes. ${ }^{36}$ Importantly, the present study was part of a larger investigation on the effect of emotion regulation on EIH. Therefore, participants included in this study received a control instruction before each exercise and the quiet condition, stating that during quiet rest and exercise, unpleasant sensations and, in case of exercises, muscle pain might occur and that they were free to cope with those sensations however they wanted.

\section{Stop-Signal Task}

A Hewlett-Packard PC (HP Inc., Palo Alto, California) with an Intel ${ }^{\circledR}$ Core $^{\text {TM }}$ i5-4590S (Intel corp., Santa Clara, CA) and an HP EliteDisplay E221c monitor with a screen size of $54.10 \mathrm{~cm}(21.50 \mathrm{inch})$ were used to run the experimental task. The stop-signal task was written by MM and administered using the Psychophysics Toolbox extensions running in MATLAB $^{\circledR}$ (MathWorks, Inc., Natick, MA) ${ }^{37}$ Participants were seated at a viewing distance of approximately $65 \mathrm{~cm}$ from the computer screen. All stimuli were displayed in the screen center and presented in black on a gray background, unless noted otherwise. Each trial began with the display of a fixation cross (0.12 inch length) for $500 \mathrm{~ms}$ (see Figure 1). Next, the target stimulus (circle: 0.9 inch $\mathrm{cm}$ in diameter; square: 0.94 inch in height and width) was presented for 1250 ms within a square-shaped frame (1.54 inches in height and width), followed by a blank screen (500 ms). On $25 \%$ of all trials (stop trial) the square-shaped frame switched to blue at a variable interval from trial onset (stop-signal delay, SSD), denoting a stop signal. On the remaining $75 \%$, the color of the square-shaped frame remained unchanged throughout the trial (go trials).

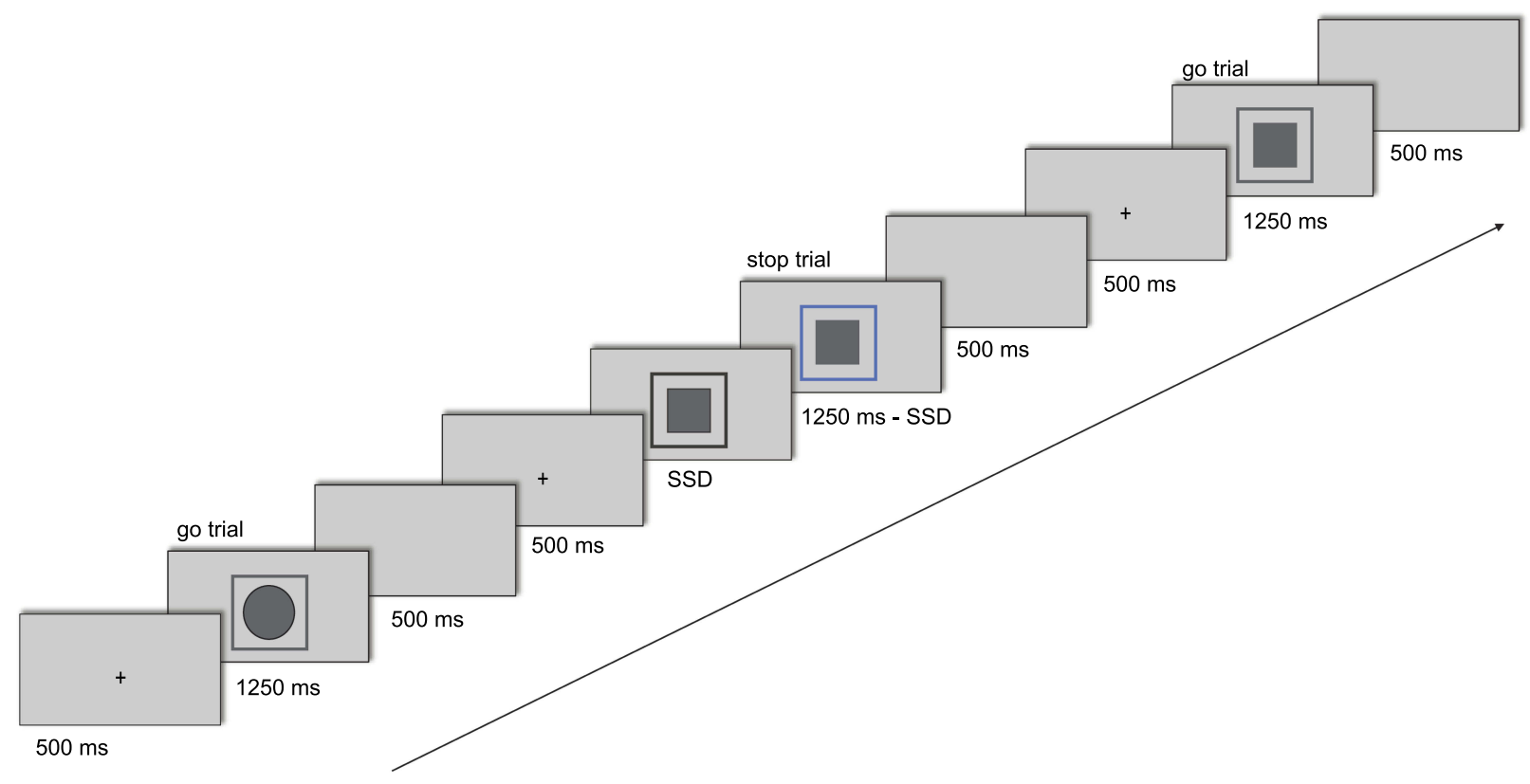

Figure I An example run of the stop-signal task, consisting of three trials. Participants' task was to indicate the target shape (circle or a square) via button presses on go trials; on stop trials, signaled by a blue frame appearing around the target shape, no response should be given. SSD denotes stop-signal delay, please see explanation in text. 
Stop and go trials were equally distributed across the two target stimuli. The initial SSD was set at $250 \mathrm{~ms}$; any subsequent SSD was determined using the one-up/onedown tracking procedure to ensure a probability of stopping of $\mathrm{P}_{\text {Respond }} \approx 0.50 .{ }^{26}$ With each response that was correctly withheld (inhibition success) the SSD was increased by $25 \mathrm{~ms}$ (maximum SSD $=1250 \mathrm{~ms}$ ). Conversely, following erroneously entered responses (inhibition failure) the SSD was decreased by $25 \mathrm{~ms}$ (minimum $\mathrm{SSD}=25 \mathrm{~ms}$ ). Participants were instructed to discriminate the target stimulus on go trials (circle vs square), indicating their decision as fast and accurately as possible by pressing the corresponding response key ("C" or "V"). On stop trials, participants were asked to refrain from responding. Participants were informed of the tracking procedure such that their probability of stopping would be maintained at about $50 \%$ for the experiment. They were therefore discouraged from waiting for stop signals to appear. Stimulus-response mapping was randomized and counterbalanced across participants.

The task was divided into four experimental blocks. In line with recommendations from Verbruggen et al, each block consisted of 64 trials (48 go trials and 16 stop trials).$^{38}$ Participants thus completed 256 trials overall for the experimental phase. An additional 32 trials were completed during the practice phase, that is, 24 go trials and 8 stop trials - again, equally divided by stimulus-type. Aside from the overall trial number, the practice phase was identical to the experimental phase in set up. During the experiment, a self-paced break was provided after 128 trials. The task lasted approximately 15 minutes.

\section{Assessment of Pain Sensitivity}

Pain sensitivity assessments, ie PPT and TSP, were conducted using a handheld algometer (Somedic Sales AB, Horby, Sweden), with a stimulation area of $1 \mathrm{~cm}^{2}$ at the thenar eminence of the non-dominant hand, hereafter termed as 'hand', and the middle of the non-dominant biceps femoris, hereafter termed as 'leg'. During assessments, participants were lying in prone position on the examination table. The order of assessment was counterbalanced and randomized for each participant but held constant throughout the individual testing sessions. Intervals of 20 seconds between assessments were maintained. All assessments were performed by female experimenters (CT and HG). For PPT assessments, the rate of pressure increase was kept to approximately $50 \mathrm{kPa} / \mathrm{sec}$. Two measurements were taken for each PPT assessment and the average of these was calculated for further analysis. ${ }^{39}$ After each PPT assessment, TSP was assessed. TSP was assessed by applying ten $\sim 1$-s repetitions of pressure at the level of the last preceding PPT, where the target force was applied as quickly as possible, with a $\sim 1$-s pause between each pulse. ${ }^{40}$ Subjects were instructed to rate the pain intensity of the first, fifth and tenth repetition on a numerical rating scale (NRS), ranging from 0 to 10 , resulting in three ratings for further analysis.

\section{Aerobic Bicycling and Isometric Knee Extension Exercises}

Participants performed a knee extension task at $30 \%$ of MVC for a duration of 90 seconds with the non-dominant leg. MVCs were obtained in the familiarization session prior to the exercise session. Participants were seated on a bench with full support of the whole thigh and 90 degrees of knee flexion. The calf of the non-dominant leg was strapped to the pillar of the bench in order to create resistance. A force transducer was placed between the strap and the shin, transducing and recording applied force in Newton (Commander Muscle Tester, Powertrack, Il, JTECH Medical, Midvale, UT, USA). The MVC was performed three times by participants by extending the knee against the resistance with maximal force. The average of the three measurements represented the individual MVC. For the exercise, participants were prepared in the same position as during the MVC assessment. They were given the display of the force transducer to continuously monitor their knee extension force of $30 \% \mathrm{MVC}$ during the task. They were instructed to hold this target force for 90 seconds.

The aerobic bicycling exercise lasted for 15 minutes. Prior to the exercise, the age-related target heart rate was determined for each subject. Based on a previously used aerobic exercise protocol demonstrating robust $\mathrm{EIH}$, a target heart rate of approximately $86 \%$ of the maximal age-related heart rate was chosen. ${ }^{41}$ This target heart rate corresponds to $75 \% \mathrm{VO}_{2 \text { max }}{ }^{42}$ Subjects performed the exercise on a stationary ergometer with a built-in heart rate monitor using a heart rate belt that was strapped around the chest (Corival cpet, Lode, Groningen, Netherlands). The subjects were asked to maintain a pedal rate of 70 rounds per minute throughout the duration of the exercise. The first two minutes of the exercise were used as a warm-up. After two minutes, resistance was increased over the next three minutes until the target heart rate was reached. The heart rate was monitored continuously, and resistance was altered in order to maintain the target 
heart rate if needed. Thirty seconds before completion of the exercise, subjects were asked to rate their level of perceived exertion due to the exercise (Borg 6-20 RPE scale). ${ }^{43}$ Furthermore, ratings of pain intensity of both exercises were obtained approx. 5 minutes after completion of the exercises on a 1-5 NRS with discrete intervals, ranging from "no pain" to "maximum pain".

\section{Statistical Analysis}

\section{Stop-Signal Task}

Data analyses were conducted using SPSS Statistics Version 25 (IBM, Armonk, NY) and R (R Core Team). ${ }^{44}$ Stop-signal reaction times (SSRTs) constituted our measure of cognitive inhibition in the statistical analyses reported below. SSRTs were estimated using the blockbased integration method. ${ }^{38}$ Specifically, SSRTs were first determined for each block separately using the integration method, as outlined in Verbruggen et al. ${ }^{45}$ To this end, for each block, the mean SSD was subtracted from the nth go RT (ie, the finishing time of the stop process). To obtain the nth go RT, all go RTs were rank-ordered and the nth go RT was identified, with $n$ constituting the total number of go trials multiplied by the probability of responding on stop trials. To illustrate, there were 48 go trials in a block; with a probability of responding on stop trials of $55 \%$, the nth go RT would then be the 26th fastest go RT in this block. The go RTs used here included correct go RTs, go omissions, which were replaced with the maximum RT, and go trials with erroneous or premature responses. Mean SSDs and the probability of responding on stop trials were, in turn, computed using the complete number of trials. The SSRTs thus estimated for a single block were then averaged across blocks to obtain the final SSRT of a given participant.

Outliers were removed in two stages. Firstly, at the individual subject level, where extreme "raw" go RT and signal-respond RT scores were removed for any one participant. Such extreme scores were identified using the threshold recommended by Leys et al (ie, median \pm 2.5 $\times$ median absolute deviation). ${ }^{46}$ On average $4 \%$ of Go trials $( \pm 4 \%)$ and $9 \%$ of signal-respond trials ( $\pm 17 \%)$ were removed at this stage. Mean go RTs were then computed for correct trials and signal-respond RTs for incorrect stop trials. Secondly, individuals exhibiting suboptimal performance were excluded at the group level, following the lenient criteria outlined by Congdon et al. ${ }^{47}$ This second stage resulted in the removal of three individuals from further analyses.

\section{Pain Sensitivity Assessment}

Temporal summation of pain (TSP) scores were calculated as the difference between the 3rd pain intensity rating (10th repetition of pressure) minus the 1 st pain intensity rating (1st repetition of pressure). Positive TSP scores thus reflect higher summation of pain ratings and negative scores a decrease in pain ratings. In order to validate the temporal summation of pain protocol, ratings of the $1 \mathrm{st}$ and the 10th repetition of pressure pulses were compared using dependent $t$-tests at each assessment site at baseline.

As both exercises were conducted within one session, a repeated-measures ANOVA was conducted in order to test if there was a change from the first to the second preexercise measurement of PPTs with the factors time (first vs second) and site (hand vs leg).

EIH absolute change scores for PPTs and TSP were calculated by subtracting pre-exercise PPT/TSP scores from post-exercise PPT/TSP scores, denoted as $\triangle \mathrm{PPT}_{\mathrm{EIH}}$ and $\triangle \mathrm{TSP}_{\mathrm{EIH}}$ hereafter. Thus, positive values of $\Delta \mathrm{PPT}_{\mathrm{EIH}}$ represent a decrease in pain sensitivity after exercise, ie, higher EIH, and, vice versa, negative values of $\Delta \mathrm{TSP}_{\mathrm{EIH}}$ represent higher EIH. Additionally, change scores in PPTs and TSP from before to after the quiet rest condition were calculated by subtracting pre-quiet rest PPT/TSP scores from post-quiet rest PPT/TSP scores, denoted as $\triangle \mathrm{PPT}_{\mathrm{QR}}$ and $\triangle \mathrm{TSP}_{\mathrm{QR}}$.

\section{Main Analysis}

Descriptive statistics are reported as means \pm standard deviations. The EIH effect was tested with dependent $t$-tests comparing $\Delta \mathrm{PPT}_{\mathrm{EIH}} / \Delta \mathrm{TSP}_{\mathrm{EIH}}$ after each exercise with $\Delta \mathrm{PPT}_{\mathrm{QR}} / \Delta \mathrm{TSP}_{\mathrm{QR}}$ after quiet rest at each assessment site, respectively. For further analysis on the association between $\mathrm{EIH}$ and cognitive inhibition, $\Delta \mathrm{PPT}_{\mathrm{EIH}} / \Delta \mathrm{TSP}_{\mathrm{EIH}}$ after each exercise were used as a measure of EIH. The association between cognitive inhibition and $\triangle \mathrm{PPT}_{\mathrm{EIH}} /$ $\Delta \mathrm{TSP}_{\mathrm{EIH}}$ after each exercise was quantified using bivariate correlation coefficients, $95 \%$ one-sided confidence intervals and with one-tailed significance testing. Spearman's rho correlation coefficients were used in case of non-normal distribution of variables. As better performance in the stop-signal task is reflected by shorter SSRTs, a negative correlation was expected in line with the hypothesis that better cognitive inhibition is associated with higher EIH. Significant correlations were then further probed using stepwise hierarchical multiple linear regression in order to control for baseline PPT and age. Homoscedasticity was inspected visually using scatterplots of standardized 
predicted values against standardized residuals, respectively. Variance inflation factors were checked regarding multicollinearity. Statistical analysis was conducted using SPSS 25 (IBM Corp, Armonk, NY) and R. ${ }^{44}$ All data were checked for plausibility and normal distribution prior to analysis. Effect sizes were calculated as Cohen's d and $r /$ rho. For Cohen's $d, d<0.20$ were considered no effect, $d=$ $0.20-0.50$ a small effect, $\mathrm{d}=0.50-0.80$ a moderate effect, and $\mathrm{d}>0.80$ a large effect. For $r /$ rho, $r /$ rho $<0.09$ were considered no effect, $r /$ rho $=0.10-0.30$ a small effect, $r /$ rho $=0.30-0.50$ a moderate effect, and $r /$ rho $>0.50$ a large effect. $^{48}$ The level of significance was set to $p=0.050$.

\section{Results}

\section{Descriptive Statistics}

\section{Participants}

Sixteen male and 21 female participants were included in the present study; mean age was $27.75 \pm 9.91$ years (mean \pm SD) within a range of 19 to 64 years, with no significant age differences between men and women $(p=0.342, \mathrm{~d}=0.48)$.

\section{Cognitive Inhibition Task}

Table 1 summarizes the descriptive statistics of stop-signal task parameters obtained in the present study. The probability of omissions and choice errors in the go-trials was very low for included participants, indicating that the participants included in the present analyses performed well on the go-trials and build up a pre-potent go-response. On stop trials, participants successfully withheld their responses on $47 \%$ of the trials, indicating that the tracking procedure was successful. The mean stop-signal reaction time (SSRT) was $261.94 \pm 37.42 \mathrm{~ms}$ (range: 189.00$326.94 \mathrm{~ms}$ ), which showed a normal distribution. Age was correlated with cognitive inhibition with a moderate effect size $(\mathrm{r}=0.39, p=0.019)$.

\section{Pressure Pain Sensitivity}

Mean pressure pain thresholds (PPT) pre quiet rest were $281.59 \pm 115.56 \mathrm{kPa}$ at the hand and $395.12 \pm 148.31 \mathrm{kPa}$ at the leg. Comparing the pain ratings of the $1^{\text {st }}$ and $10^{\text {th }}$ pressure pulses in the temporal summation of pain protocol pre quiet rest yielded significant increases in pain ratings after repeated stimulation at each assessment site (all $\mathrm{p}<0.001$ ), validating the TSP protocol. TSP scores pre quiet rest were $2.32 \pm 1.65$ at the hand, and $2.13 \pm 1.36$ at the leg.

Comparing pre exercise PPTs from the first exercise to the second exercise using a $2 \times 2 \mathrm{rm}$ ANOVA with the factors time (first vs second exercise) and site (hand vs leg) yielded no significant main effect of time, $F(1,36)=$ $0.843, p=0.365, \eta_{\mathrm{p}}{ }^{2}=0.02$, suggesting that pre-exercise PPTs remained stable from before the first to before the second exercise.

\section{Exercise Parameters}

The aerobic bicycling exercise was performed with a mean target heart rate of $163.11 \pm 9.71$ beats/minute, which corresponded to $85.9 \%$ of the age-related maximum heart rate and resulted in a mean Borg scale value of $15.70 \pm$ 1.41. Participants rated the aerobic bicycling exercise with a painfulness of $2.21 \pm 1.08$ and an unpleasantness of 3.00 \pm 1.10 on a $1-5$ NRS. In the isometric knee extension task, participants performed with a mean target force of $60.77 \pm$ $29.43 \mathrm{~N}$, which corresponded to $30 \%$ of the MVC. The knee extension exercise was rated with a painfulness of $1.89 \pm 0.94$ and an unpleasantness of $1.91 \pm 1.06$.

Table I Mean \pm SD and Range for the Main Dependent Measures Directly Observed in the Stop-Signal Task, Reported Separately for Participants Included in (versus Excluded from) Subsequent Analyses

\begin{tabular}{|l|l|l|l|l|}
\hline \multirow{2}{*}{} & \multicolumn{2}{l|}{ Included Participants (N = 37) } & \multicolumn{2}{l|}{ Excluded Participants (N = 3) } \\
\cline { 2 - 5 } & Mean \pm SD & Range & Mean \pm SD & Range \\
\hline Probability of go omissions (no response) & $0.01 \pm 0.02$ & $0-0.06$ & $0.12 \pm 0.14$ & $0-0.27$ \\
Probability of choice errors on go trials & $0.02 \pm 0.02$ & $0-0.07$ & $0.48 \pm 0.44$ & $0.11-0.97$ \\
RT on go trials (mean) & $592.67 \pm 172.91$ & $394.46-991.47$ & $825.15 \pm 253.33$ & $544.88-1037.82$ \\
Intra-subject variability of correct go trials & $103.25 \pm 32.33$ & $53.59-165.78$ & $154.30 \pm 46.65$ & $100.44-181.73$ \\
Probability of responding on a stop trial & $0.47 \pm 0.06$ & $0.34-0.58$ & $0.38 \pm 0.09$ & $0.30-0.47$ \\
Average stop-signal delay & $314.17 \pm 148.29$ & $91.41-638.28$ & $482.55 \pm 185.83$ & $279.69-644.53$ \\
Stop-signal reaction time & $261.94 \pm 34.73$ & $189.00-326.94$ & - & - \\
RT of go responses on unsuccessful stop trials & $518.79 \pm 149.68$ & $355.47-882.73$ & $680.29 \pm 213.69$ & $435.73-831.01$ \\
\hline
\end{tabular}

Notes: In line with the recommendations by Verbruggen et al ${ }^{45}$, SSRTs were not estimated for participants with sub-optimal task performance (here: excluded participants). 


\section{Exercise-Induced Hypoalgesia}

After the aerobic bicycling exercise, there was a mean percentage change in PPTs of $4.73 \pm 21.08 \%$ at the hand and $8.00 \pm 16.80 \% . \Delta \mathrm{PPT}_{\mathrm{EIH}}$ after aerobic exercise differed from $\triangle \mathrm{PPT}_{\mathrm{QR}}$ after quiet rest at the hand $(\mathrm{d}=0.52, p=0.034)$ and at the leg $(\mathrm{d}=0.64, p=0.010)$, with moderate effect sizes. After isometric knee extension, there was a mean percentage change in PPTs of $0.94 \pm 14.68 \%$ at the hand and a mean percentage change in PPTs of $4.62 \pm 19.15 \%$ at the leg. No significant difference in $\triangle \mathrm{PPT}_{\mathrm{EIH}}$ after isometric exercise and $\triangle \mathrm{PPT}_{\mathrm{QR}}$ was observed at the hand $(\mathrm{d}=0.38, p=0.136)$, nor at the leg $(\mathrm{d}=0.42, p=0.137)$ with small effect sizes. No effects of the exercises were seen on TSP scores at any assessment site (see Table 2). Notably, there were decreases in PPTs following the quiet rest condition, which were comparable to the increases in PPTs after aerobic exercise.

Absolute change scores in pressure pain thresholds $\left(\triangle \mathrm{PPT}_{\mathrm{EIH}}\right)$ and temporal summation of pain scores $\left(\Delta \mathrm{TSP}_{\mathrm{EIH}}\right)$, as well as absolute change scores for quiet rest $\left(\Delta \mathrm{PPT}_{\mathrm{QR}} / \Delta \mathrm{TSP}_{\mathrm{QR}}\right)$, each at the hand and at the leg, are shown in Table 2.

\section{Association Between Cognitive Inhibition and $\mathrm{EIH}$}

A significant, moderate negative correlation was observed between SSRT and $\triangle$ PPTs at the hand, after aerobic bicycling ( $r=-0.35,95 \% \mathrm{CI}:-0.57 ;-0.08, p=0.021)$. This means that shorter SSRTs, ie, better cognitive inhibition performance, were associated with higher widespread EIH after aerobic bicycling (Figure 2). No other correlations were of an effect size beyond small or no effect, had a 95\% confidence interval below zero, and $p$-values were far from the threshold of significance (see Table 3). Scatterplots for all correlations are displayed in Figures S1-S7.

Hierarchical multiple linear regression with $\triangle \mathrm{PPT}$ after aerobic exercise at the hand resulted in a final model with SSRT as the only significant predictor, $\beta=-0.33 ; \mathrm{R}^{2}=0.11$, adjusted $\mathrm{R}^{2}=$ 0.08 , with a significant change in $\mathrm{F}$ from mean, $p=0.048$. None of the control variables at baseline PPT and age reached significance and were therefore excluded from the final model. Inspecting Cook's distances, no influential cases were identified. Residuals were independent (Durbin Watson $=2.22$ ) and

Table 2 Change Scores $(\Delta)$, Displayed in Mean \pm SD for PPT and TSP from Before to After Aerobic Bicycling and Isometric Knee Exercises, as Well as the Quiet Rest Control Condition, as Assessed at the Hand and the Leg with Significance and Effect Sizes (Cohen's d) for Tests of the Difference Between Exercises and Quiet Rest

\begin{tabular}{|c|c|c|c|c|}
\hline \multicolumn{5}{|c|}{ Aerobic Bicycling } \\
\hline & $\begin{array}{l}\triangle \mathrm{PPT}_{\mathrm{EIH}} \\
(\mathrm{kPa})\end{array}$ & & $\Delta T_{S P} P_{E I H}(N R S I-10)$ & \\
\hline Hand & $7.24 \pm 54.60^{\mathrm{a}}$ & $\begin{array}{l}p=0.034 \\
d=0.52\end{array}$ & $0.35 \pm 1.32^{b}$ & $\begin{array}{l}p=0.453 \\
d=0.19\end{array}$ \\
\hline Leg & $35.23 \pm 75.22^{b}$ & $\begin{array}{l}p=0.017 \\
d=0.64\end{array}$ & $-0.24 \pm 1.44^{b}$ & $\begin{array}{l}p=0.332 \\
d=0.27\end{array}$ \\
\hline \multicolumn{5}{|c|}{ Isometric Knee Extension } \\
\hline Hand & $-2.83 \pm 41.38^{\mathrm{a}}$ & $\begin{array}{l}p=0.136 \\
d=0.38\end{array}$ & $0.22 \pm 1.36^{b}$ & $\begin{array}{l}p=0.624 \\
d=0.10\end{array}$ \\
\hline Leg & $20.68 \pm 79.50^{b}$ & $\begin{array}{l}p=0.137 \\
d=0.42\end{array}$ & $0.22 \pm 1.06^{b}$ & $\begin{array}{l}p=0.449 \\
d=0.10\end{array}$ \\
\hline \multicolumn{5}{|c|}{ Quiet Rest } \\
\hline & $\begin{array}{l}\triangle P P T_{Q R} \\
(\mathrm{kPa})\end{array}$ & & $\begin{array}{l}\triangle \mathrm{PPT}_{\mathrm{QR}} \\
(\mathrm{NRS} \mathrm{I}-10)\end{array}$ & \\
\hline $\begin{array}{l}\text { Hand } \\
\text { Leg }\end{array}$ & $\begin{array}{l}-21.16 \pm 54.24^{a} \\
-10.66 \pm 68.17^{a}\end{array}$ & - & $\begin{array}{l}0.11 \pm 1.10^{\mathrm{b}} \\
0.11 \pm 1.4 \mathrm{I}^{\mathrm{b}}\end{array}$ & $\begin{array}{l}- \\
-\end{array}$ \\
\hline
\end{tabular}

Notes: for $\triangle \mathrm{PPT}$, positive values indicate a decrease in pain sensitivity (ie ElH), and negative values indicate an increase in pain sensitivity (ie hyperalgesia after exercise), while the opposite is true for $\Delta \mathrm{TSP}$, ie positive values indicate an increase in pain sensitivity (ie hyperalgesia after exercise). $P$-values and Cohen's $\mathrm{d}$ effect sizes refer to the test of difference between change scores in the respective exercise vs quiet rest condition (eg $\triangle \mathrm{PPT}_{\mathrm{EIH}}$ after aerobic bicycling at the hand vs $\Delta \mathrm{PPT}$ QR at the hand). ${ }^{\mathrm{N}} \mathrm{Normally}$ distributed; $t$-test for dependent samples to test the difference between exercise and quiet rest. ${ }^{b}$ Not-normally distributed; Wilcoxon-rank-sum-test for dependent samples to test the difference between exercise and quiet rest. 


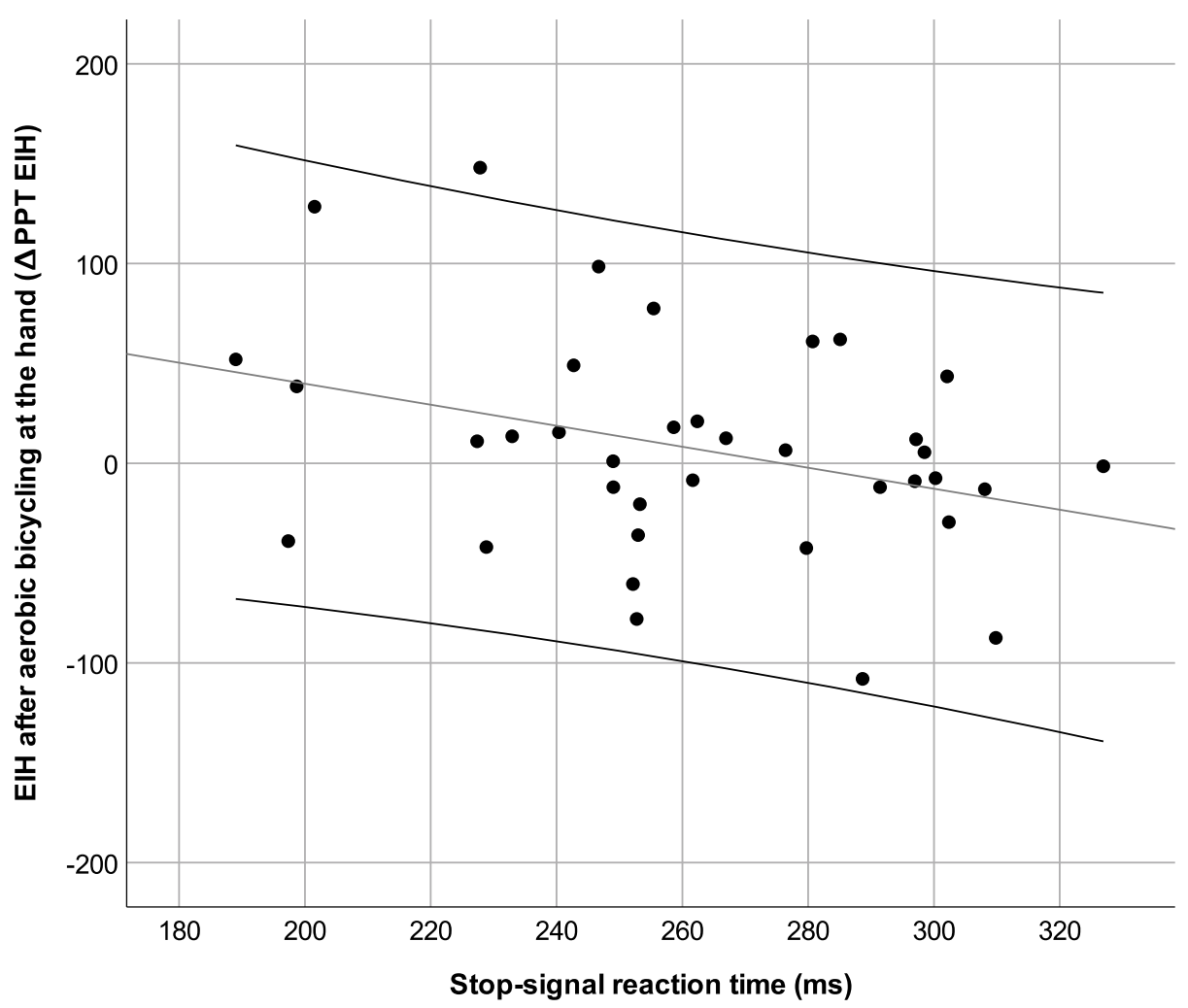

Figure 2 Scatterplot of $\triangle \mathrm{PPT}$ after aerobic bicycling exercise at the hand and stop-signal reaction times, depicting a moderate negative correlation of $(r=-0.35,95 \% \mathrm{Cl}:-0.57$; $-0.08, p=0.02 \mathrm{I})$. Note that shorter stop-signal reaction times represent better cognitive inhibition. Scatterplots for all other correlations are displayed in Figures SI-S7.

normally distributed, as indicated by Kolmogorov-Smirnov tests of standardized residuals $(p>0.200)$ in this model. Homoscedasticity was marginally given, as inspected by scatterplots of standardized predicted values against standardized residuals. Variance inflation factors indicated that multicollinearity was not an issue (VIF $=1.06-1.18)$.

\section{Discussion}

The present study investigated the association between cognitive inhibition and exercise-induced hypoalgesia (EIH) after two different types of exercises. We observed an association between better cognitive inhibition and higher EIH effect after aerobic bicycling at the hand. EIH was observed after aerobic bicycling, but unexpectedly, no EIH was observed after the isometric knee extension.

\section{Positive Association Between Cognitive Inhibition and Widespread ElH}

Partly confirming the hypothesis, there was a moderate correlation between performance in the cognitive inhibition task and

Table 3 Correlation Coefficients with $95 \%$ Confidence Intervals $(95 \% \mathrm{Cl})$ and p-values Between Stop-Signal Reaction Times (SSRT) and $\triangle \mathrm{PPT}$ and $\triangle \mathrm{TSP}$ for Each Exercise and Assessment Site. Scatterplots for Each Correlation are Displayed in Figures SI-S7

\begin{tabular}{|c|c|c|c|c|c|c|}
\hline & \multicolumn{3}{|l|}{$\Delta \mathbf{P P T}_{\text {EIH }}$} & \multicolumn{3}{|l|}{$\Delta \mathbf{T S P}_{\mathrm{EIH}}$} \\
\hline & Coefficient & 95\% Cl & $p$ & Coefficient & $95 \% \mathrm{Cl}$ & $p$ \\
\hline \multirow{3}{*}{$\begin{array}{l}\text { Hand } \\
\text { Leg }\end{array}$} & \multicolumn{6}{|c|}{ Aerobic Bicycling } \\
\hline & $r=-0.35$ & $-0.57,-0.08$ & 0.021 & rho $=-0.14$ & $-0.40,0.14$ & 0.397 \\
\hline & rho $=-0.10$ & $-0.36,0.18$ & 0.553 & rho $=0.02$ & $-0.26,0.29$ & 0.904 \\
\hline \multirow{3}{*}{$\begin{array}{l}\text { Hand } \\
\text { Leg }\end{array}$} & \multicolumn{6}{|c|}{ Isometric Knee Extension } \\
\hline & $r=-0.03$ & $-0.30,0.25$ & 0.884 & rho $=-0.09$ & $-0.36,0.20$ & 0.598 \\
\hline & rho $=0.03$ & $-0.25,0.30$ & 0.857 & rho $=-0.06$ & $-0.33,0.22$ & 0.728 \\
\hline
\end{tabular}


EIH at the hand after aerobic bicycling. The correlation between cognitive inhibition and EIH is in line with previous studies, reporting that higher cognitive inhibition is associated with conditioned pain modulation (CPM) and higher pain tolerance, as well as lower pain threshold. ${ }^{12,19,28,29,49,50}$

Cognitive inhibition depends on the neurophysiological integrity of the prefrontal cortex (PFC) and the anterior cingulate cortex (ACC), demonstrating a noteworthy overlap with brain structures implied in CPM and other forms of pain inhibition, such as distraction. ${ }^{21,31,32}$ This suggests that cognitive inhibition might be a neurocognitive aspect in pain inhibition. ${ }^{12}$ In the psychological literature, extensive evidence indicates that cognitive inhibition determines the efficiency of emotion regulation, which refers to the process of down-regulation of aversive affective states. $^{51,52}$ A similar relationship has been proposed for cognitive inhibition and pain inhibition, as the latter can be conceived as a form of emotion regulation. ${ }^{17,51,53}$ This stands to reason considering that emotion regulation strategies known from psychological research informed the investigation of now well-researched pain regulation strategies, such as distraction. ${ }^{54}$ Distraction, in turn, was proposed as a psychological mediator of $\mathrm{EIH}$, as during exercise, the attention might be directed towards somatic sensations of sweating or heart pounding, and away from pain. ${ }^{55}$ Thus, it might be that better cognitive inhibition abilities support the efficiency of cognitive pain regulation strategies during exercise that might in turn lead to more EIH.

An association with cognitive inhibition was only observed for the change of PPTs after aerobic exercise at the hand, partly confirming the hypothesis. However, no association was observed for change in PPTs after aerobic exercise at the leg, nor with change in PPTs after isometric exercise at any assessment site, nor with any of the PPT scores. These results considerably limit the strength of the conclusion in this study and warrant explanation. It has been proposed before that widespread EIH that is assessed at sites remote from the exercising muscle, are due to the systemic activation of central pain inhibitory mechanisms. ${ }^{56}$ On the other hand, local EIH assessed at the exercising muscle has been proposed to reflect a superimposition of central pain inhibition and peripheral processes in the muscle tissue. Concurrently, cognitive inhibition might influence central pain inhibition, ie, widespread EIH rather than local, as widespread $\mathrm{EIH}$ and cognitive inhibition likely emerge in the central nervous system. ${ }^{21,33}$ Therefore, cognitive inhibition might be more strongly related to widespread $\mathrm{EIH}$ assessed at remote sites than to local EIH assessed at the exercising muscles. This might explain the null findings on the correlations between cognitive inhibition and $\mathrm{EIH}$ assessed at the leg, as this assessment site was close to the exercising muscles. However, the biceps femoris is likely not involved in bicycling or knee extension. Therefore, it is not possible to make definite inferences about the influence of local processes on the association between cognitive inhibition and $\mathrm{EIH}$ in the present study. Therefore, future studies should systematically investigate the association between cognitive inhibition and EIH with a distinction between local and remote assessment sites.

In sum, this study provides preliminary evidence for an association between cognitive inhibition and EIH after aerobic exercise. In synopsis with previous research, these results imply that cognitive inhibition might be a specific neurocognitive aspect in the regulation of pain during exercise. Lastly, the influence of a truly local assessment site on the association between cognitive inhibition and EIH should be investigated in future studies.

\section{Absence of a Distinct ElH Effect After Isometric Knee Extension}

There was a small and moderate effect size for EIH after isometric knee extension at the hand and at the leg, respectively. However, these effects did not reach significance, suggesting that EIH was not successfully induced after isometric exercise. As previous studies report robust EIH after submaximal isometric knee extension of a similar duration, this result was unexpected and warrants explanation. ${ }^{36,57-60}$ As only local $\mathrm{EIH}$ after submaximal isometric exercises has been reported, the absence of a truly local assessment site might account for this finding. ${ }^{61}$ Furthermore, the rating of painfulness of the isometric knee extension exercise was low compared to the rating of aerobic bicycling exercise. Painful exercises have been reported to elicit higher EIH responses than non-painful exercises. $^{62}$ Therefore, the low painfulness of the knee extension exercise in this study might be another explanation for diminished EIH after isometric knee extension in this study.

\section{Temporal Summation of Pain (TSP)}

No effects of exercise were seen on TSP in the present study. This is in contrast to studies reporting effects of exercise on TSP induced using computer-controlled heat pain $^{60,61}$ or computer-controlled cuff pressure pain. ${ }^{62,63}$ The current study employed a TSP protocol using manual algometry. Although TSP induced with manual pressure algometry appears to be reliable, it might be that this type of TSP is not sensitive to exercise. ${ }^{63}$ 


\section{Clinical Implications}

Although pain-free, healthy participants took part in the present study, we believe that the present results bear clinical potential. It has been proposed that dysfunctional EIH might cause pain exacerbation after exercise in a subset of patients with chronic pain and thus promote low adherence and intolerance to exercise interventions. ${ }^{4}$ Concurrently, cognitive inhibition has been observed to be reduced in chronic pain, in line with reduced efficiency of pain regulation by means of cognitive strategies. ${ }^{13,53,54} \mathrm{~A}$ link between cognitive inhibition and EIH could imply that a gain of function in cognitive inhibition can transfer into a gain of function in EIH. Concurrently, cognitive inhibition has been shown to be improved by means of neuropsychological training and mindfulness meditation. ${ }^{64,65}$ Therefore, this link might provide additional, low-threshold training options in chronic pain conditions in which exercise has become aversive due to learning history. Therefore, future studies should investigate whether the link between cognitive inhibition and widespread EIH reported here translates into chronic pain.

\section{Limitations}

Although a causal link between cognitive inhibition and descending pain inhibition is supported by the literature, the correlational design of this study does not allow for the causal interpretations proposed in the discussion. ${ }^{12,19,50}$ Therefore, the present interpretations should be cautiously viewed as stimulation for future research and be validated in clinical samples with chronic pain. Furthermore, this study faces some other limitations that limits it's generalizability. Furthermore, the present sample was a control group for a larger experimental investigation on pain regulation strategies pertaining to EIH. This study was an exploratory pilot conducted within another study of broader scope, where multiple other measurements, such as blood pressure and salivary cortisol, were assessed along with pain sensitivity. Since an influence of these measures cannot be ruled out, the current study is in need of replication.

Additionally, participants in the present study received a control instruction before the exercises and quiet rest, which mentioned painful and unpleasant sensations that might occur during exercise or quiet rest. It is possible that cognitive inhibition plays less of a role in EIH if such an intervention is omitted. Furthermore, the instruction might account for the decrease in pain sensitivity observed after quiet rest. Lastly, the duration of the 15-min quiet rest in the EIH paradigm was matched to the 15-min aerobic bicycling exercise, but not the
$90 \mathrm{sec}$ isometric knee extension, limiting the direct comparability between quiet rest and isometric knee extension.

Related to this, a power analysis for the investigated effect was not conducted in advance of the study. A posteriori power analysis with a correlational effect size of $r=0.35$ indicates that at least 63 participants are needed to achieve a power of $p=0.90$. Therefore, although the present study was sufficiently powered for large EIH effects, it was not sufficiently powered for the correlation between cognitive inhibition and EIH, as the present and previous results suggest a small to moderate effect size. ${ }^{3,19,48}$ This is all the more so as both EIH effects and stop-signal response times have been reported to show low reliability, further compromising the power and validity of correlational analysis. ${ }^{24,27,66,67}$

Another clear limitation of this study is the absence of a truly local assessment site for EIH after both exercises, which results in two considerable limitations: firstly, this might be the reason for the absence of a clear EIH effect after the isometric knee extension, as outlined in 4.2; secondly, as explicated in 4.1, it limits any inference about whether local processes might have weakened the association between cognitive inhibition and EIH in this study.

It was decided out of practical considerations to employ only one type of neuropsychological task to assess cognitive inhibition. However, it is well recognized that different cognitive inhibition tasks assess different sub-aspects of inhibition. ${ }^{24}$ Also, any cognitive task assumed to be measuring executive functions requires a number of different processes in addition to the targeted executive function, such as motor and sensory aspects, resulting in task impurity. ${ }^{23}$ Both of these issues restrict the validity of the interpretations regarding the specific function of cognitive inhibition. This may be addressed in future studies by 1) employing a comprehensive assessment with different executive functioning tasks and using latent variable analysis to extract a common factor representing cognitive inhibition and 2) employing tasks optimal for diffusion model analyses to decompose the underlying cognitive processes, which would also improve the reliability of the obtained measures. ${ }^{23,68}$

\section{Conclusion}

This is the first study to report an association between cognitive inhibition performance and exercise-induced hypoalgesia after aerobic bicycling. This finding fits well with previous studies reporting a link between cognitive inhibition and experimental pain measures of descending pain inhibition and is potential of clinical importance. However, no EIH was observed after isometric exercise. 


\section{Acknowledgments}

We acknowledge support by the DFG Open Access

Publication Funds from Ruhr University Bochum.

\section{Protocol Reference}

dx.doi.org/10.17504/protocols.io.873hzqn.

\section{Disclosure}

The authors declare that they have no conflict of interest.

\section{References}

1. Ossipov MH, Morimura K, Porreca F. Descending pain modulation and chronification of pain. Curr Opin Support Palliat Care. 2014;8 (2):143-151. doi:10.1097/SPC.0000000000000055

2. Yarnitsky D. Role of endogenous pain modulation in chronic pain mechanisms and treatment. Pain. 2015;156(Suppl 1):S24-31. doi:10.1097/01.j.pain.0000460343.46847.58

3. Naugle KM, Fillingim RB, Riley JL. A meta-analytic review of the hypoalgesic effects of exercise. J Pain. 2012;13(12):1139-1150. doi:10.1016/j.jpain.2012.09.006

4. Rice D, Nijs J, Kosek E, et al. Exercise-induced hypoalgesia in pain-free and chronic pain populations: state of the art and future directions. J Pain. 2019;20(11):1249-1266. doi:10.1016/j.jpain.2019.03.005

5. Vaegter HB, Lyng KD, Yttereng FW, Christensen MH, Sørensen MB, Graven-Nielsen T. Exercise-induced hypoalgesia after isometric wall squat exercise: a test-retest reliability study. Pain Med. 2019a;20 (1):129-137. doi:10.1093/pm/pny087

6. Ohlman T, Miller L, Naugle KE, Naugle KM. Physical activity levels predict exercise-induced hypoalgesia in older adults. Med Sci Sports Exerc. 2018;50(10):2101-2109. doi:10.1249/MSS.0000000000001661

7. Awali A, Nevsimal R, O'Melia S, Alsouhibani A, Bement MH. The contribution of experimental pain to the sex differences in exerciseinduced hypoalgesia. J Pain. 2019;20(4):S50-S50.

8. Peterson JA, Schubert DJ, Campbell J, Bemben MG, Black CD. Endogenous pain inhibitory function: endurance-trained athletes vs active controls. Pain Med. 2019;20(9):1822-1830. doi:10.1093/pm/pnz014

9. Smith A, Ritchie C, Pedler A, McCamley K, Roberts K, Sterling M. Exercise induced hypoalgesia is elicited by isometric, but not aerobic exercise in individuals with chronic whiplash associated disorders Scand J Pain. 2017;15(1):14-21. doi:10.1016/j.sjpain.2016.11.007

10. Brellenthin AG, Cook D, Sehgal N, Koltyn KF. Exercise-induced hypoalgesia and pain catastrophizing in women and men. Med Sci Sports Exerc. 2013;45(5):280.

11. Naugle KM, Naugle KE, Fillingim RB, Riley JL. Isometric exercise as a test of pain modulation: effects of experimental pain test, psychological variables, and sex. Pain Med. 2014a;15(4):692-701. doi:10.1111/pme.12312

12. Bjekić J, Živanović M, Purić D, Oosterman JM, Filipović SR. Pain and executive functions: a unique relationship between Stroop task and experimentally induced pain. Psychol Res. 2018;82(3):580-589. doi:10.1007/ s00426-016-0838-2

13. Berryman C, Stanton TR, Bowering KJ, Tabor A, McFarlane A, Moseley GL. Do people with chronic pain have impaired executive function? A meta-analytical review. Clin Psychol Rev. 2014;34 (7):563-579. doi:10.1016/j.cpr.2014.08.003

14. Staud R. Abnormal endogenous pain modulation is a shared characteristic of many chronic pain conditions. Expert Rev Neurother. 2012;12(5):577-585. doi:10.1586/ern.12.41

15. MacPherson SE, Phillips LH, Della Sala S. Age, executive function and social decision making: a dorsolateral prefrontal theory of cognitive aging. Psychol Aging. 2002;17(4):598-609. doi:10.1037/0882-7974.17.4.598
16. Hermans L, van Oosterwijck J, Goubert D, et al. Inventory of personal factors influencing conditioned pain modulation in healthy people: a systematic literature review. Pain Pract. 2016;16(6):758-769. doi:10.1111/papr.12305

17. Verhoeven K, van Damme S, Eccleston C, van Ryckeghem DML, Legrain V, Crombez G. Distraction from pain and executive functioning: an experimental investigation of the role of inhibition, task switching and working memory. Eur J Pain. 2011;15(8):866-873. doi:10.1016/j.ejpain.2011.01.009

18. Buhle JT, Wager TD. Performance-dependent inhibition of pain by an executive working memory task. Pain. 2010;149(1):19-26. doi:10. 1016/j.pain.2009.10.027

19. Marouf R, Caron S, Lussier M, Bherer L, Piché M, Rainville P. Reduced pain inhibition is associated with reduced cognitive inhibition in healthy aging. Pain. 2014;155(3):494-502. doi:10.1016/j. pain.2013.11.011

20. Miyake A, Friedman NP. The nature and organization of individual differences in executive functions: four general conclusions. Curr Dir Psychol Sci. 2012;21(1):8-14. doi:10.1177/0963721411429458

21. Aron AR. The neural basis of inhibition in cognitive control. Neuroscientist. 2007;13(3):214-228. doi:10.1177/1073858407299288

22. Bari A, Robbins TW. Inhibition and impulsivity: behavioral and neural basis of response control. Prog Neurobiol. 2013;108:44-79. doi:10.1016/j.pneurobio.2013.06.005

23. Snyder HR, Miyake A, Hankin BL. Advancing understanding of executive function impairments and psychopathology: bridging the gap between clinical and cognitive approaches. Front Psychol. 2015;6:328. doi:10.3389/fpsyg.2015.00328

24. Friedman NP, Miyake A. Unity and diversity of executive functions: individual differences as a window on cognitive structure. Cortex. 2017;86:186-204. doi:10.1016/j.cortex.2016.04.023

25. Garavan H, Hester R, Murphy K, Fassbender C, Kelly C. Individual differences in the functional neuroanatomy of inhibitory control. Brain Res. 2006;1105(1):130-142. doi:10.1016/j.brainres.2006.03.029

26. Matzke D, Verbruggen F, Logan GD. The stop-signal paradigm. In: Wixted JT, Stevens SS, editors. Stevens' Handbook of Experimental Psychology and Cognitive Neuroscience. Vol. 6, Fourth. New York: John Wiley \& Sons, Inc;2018:1-45

27. Hedge C, Powell G, Sumner P. The reliability paradox: why robust cognitive tasks do not produce reliable individual differences. Behav Res Methods. 2018;50(3):1166-1186. doi:10.3758/s13428017-0935-1

28. Oosterman JM, Dijkerman HC, Kessels RPC, Scherder EJA. A unique association between cognitive inhibition and pain sensitivity in healthy participants. Eur J Pain. 2010;14(10):1046-1050. doi:10. 1016/j.ejpain.2010.04.004

29. Coppieters I, Ickmans K, Cagnie B, et al. Cognitive performance is related to central sensitization and health-related quality of life in patients with chronic whiplash-associated disorders and fibromyalgia. Pain Physician. 2015;18(3):E389-401.

30. Valet M, Sprenger T, Boecker H, et al. Distraction modulates connectivity of the cingulo-frontal cortex and the midbrain during painan fMRI analysis. Pain. 2004;109(3):399-408. doi:10.1016/j.pain.20 04.02 .033

31. Knudsen L, Petersen GL, Nørskov KN, et al. Review of neuroimaging studies related to pain modulation. Scand J Pain. 2018;2 (3):108-120. doi:10.1016/j.sjpain.2011.05.005

32. Bantick SJ, Wise RG, Ploghaus A, Clare S, Smith SM, Tracey I. Imaging how attention modulates pain in humans using functional MRI. Brain. 2002;125(2):310-319. doi:10.1093/brain/awf022

33. Scheef L, Jankowski J, Daamen M, et al. An fMRI study on the acute effects of exercise on pain processing in trained athletes. Pain. 2012;153(8):1702-1714. doi:10.1016/j.pain.2012.05.008

34. Black GAR, Renfroe JB, Hight RE. The effects of caffeine ingestion on exercise-induced hypoalgesia: a pilot study. Physiol Behav. 2016b;161:1-6. doi:10.1016/j.physbeh.2016.04.005 
35. Verbruggen F, Logan GD. Response inhibition in the stop-signal paradigm. Trends Cogn Sci. 2008;12(11):418-424. doi:10.1016/j.tics.2008.07.005

36. Vaegter HB, Handberg G, Graven-Nielsen T. Similarities between exercise-induced hypoalgesia and conditioned pain modulation in humans. Pain. 2014a;155(1):158-167. doi:10.1016/j.pain.2013.09.023

37. Kleiner M, Brainard DH, Pelli D, Ingling A, Murray R, Broussard C. What's new in psychtoolbox-3. Perception. 2007;36(4):1-16.

38. Verbruggen F, Chambers CD, Logan GD. Fictitious inhibitory differences: how skewness and slowing distort the estimation of stopping latencies. Psychol Sci. 2013;24(3):352-362. doi:10.1177/0956797612457390

39. Cathcart S, Pritchard D. Reliability of pain threshold measurement in young adults. J Headache Pain. 2006;7(1):21-26. doi:10.1007/ s10194-006-0265-7

40. Vaegter HB, Handberg G, Graven-Nielsen T. Isometric exercises reduce temporal summation of pressure pain in humans. Eur $J$ Pain. 2014b;19(7):973-983. doi:10.1002/ejp.623

41. Vaegter HB, Handberg G, Jorgensen MN, Kinly A, Graven-Nielsen T. Aerobic exercise and cold pressor test induce hypoalgesia in active and inactive men and women. Pain Med. 2015;16(5):923-933. doi:10.1111/pme.12641

42. Swain DP, Abernathy KS, Smith CS, Lee SJ, Bunn SA. Target heart rates for the development of cardiorespiratory fitness. Med Sci Sports Exerc. 1994;26(1):112-116. doi:10.1249/00005768-199401000-00019

43. Borg GA. Psychophysical bases of perceived exertion. Med Sci Sports Exerc. 1982;14(5):377-381. doi:10.1249/00005768-198205000-00012

44. R Core Team. R: A Language and Environment for Statistical Computing. Vienna, Austria: R Foundation for Statistical Computing; 2013. Available from: http://www.R-project.org/. Accessed April 14, 2020.

45. Verbruggen F, Aron AR, Band GP, et al. A consensus guide to capturing the ability to inhibit actions and impulsive behaviors in the stop-signal task. Elife. 2019;8. doi:10.7554/eLife.46323

46. Leys C, Ley C, Klein O, Bernard P, Licata L. Detecting outliers: do not use standard deviation around the mean, use absolute deviation around the median. J Exp Soc Psychol. 2013;49(4):764-766. doi:10.1016/j.jesp.2013.03.013

47. Congdon E, Mumford JA, Cohen JR, Galvan A, Canli T, Poldrack RA. Measurement and reliability of response inhibition. Front Psychol. 2012;3:37. doi:10.3389/fpsyg.2012.00037

48. Cohen JL. Statistical Power Analysis for the Behavioral Sciences. Hillsday, NJ: Erlbaum; 1988.

49. Karsdorp PA, Geenen R, Vlaeyen JWS. Response inhibition predicts painful task duration and performance in healthy individuals performing a cold pressor task in a motivational context. Eur J Pain. 2014;18 (1):92-100. doi:10.1002/j.1532-2149.2013.00348.x

50. Zhou S, Kemp J, Després O, Pebayle T, Dufour A. The association between inhibition and pain tolerance in the elderly: evidence from event-related potentials. Eur J Pain. 2015;19(5):669-676. doi:10.1002/ejp.588

51. Hofmann W, Schmeichel BJ, Baddeley AD. Executive functions and self-regulation. Trends Cogn Sci. 2012;16(3):174-180. doi:10.1016/j. tics.2012.01.006

52. Ochsner KN, Gross JJ. The cognitive control of emotion. Trends Cogn Sci. 2005;9(5):242-249. doi:10.1016/j.tics.2005.03.010

53. Solberg Nes L, Roach AR, Segerstrom SC. Executive functions, selfregulation, and chronic pain: a review. Ann Behav Med. 2009;37 (2):173-183. doi:10.1007/s12160-009-9096-5
54. van Ryckeghem DM, van Damme S, Eccleston C, Crombez G. The efficacy of attentional distraction and sensory monitoring in chronic pain patients: a meta-analysis. Clin Psychol Rev. 2018;59:16-29. doi:10.1016/j.cpr.2017.10.008

55. Nijs J, Kosek E, van Oosterwijck J, Meeus M. Dysfunctional endogenous analgesia during exercise in patients with chronic pain: to exercise or not to exercise? Pain Physician. 2012;15(3 Suppl):ES205-13.

56. Micalos PS. Perspectives on biochemical and neurosensory mechanisms for exercise-induced pain inhibition. Fatigue. 2014;2(4):219230. doi:10.1080/21641846.2014.957484

57. Kosek E, Lundberg L. Segmental and plurisegmental modulation of pressure pain thresholds during static muscle contractions in healthy individuals. Eur J Pain. 2003;7(3):251-258. doi:10.1016/S1090-3801 (02)00124-6

58. Lannersten L, Kosek E. Dysfunction of endogenous pain inhibition during exercise with painful muscles in patients with shoulder myalgia and fibromyalgia. Pain. 2010;151(1):77-86. doi:10.1016/j.pain.20 10.06.021

59. Tour J, Lofgren M, Mannerkorpi K, et al. Gene-to-gene interactions regulate endogenous pain modulation in fibromyalgia patients and healthy controls-antagonistic effects between opioid and serotoninrelated genes. Pain. 2017;158(7):1194-1203. doi:10.1097/j.pain.000 0000000000896

60. Koltyn KF. Exercise-induced hypoalgesia and intensity of exercise. Sports Med. 2002;32(8):477-487. doi:10.2165/00007256-2002320 80-00001

61. Alsouhibani A, Hrdina E, Krajewski R, Chirayil A, Stolzman S, Bement MH. (465) Exercise-induced hypoalgesia following six minute walk test. J Pain. 2016;17(4):S90-S90. doi:10.1016/j.jpain.20 16.01 .442

62. Ellingson LD, Cook DB. The hypoalgesic effects of exercise: characteristics, mechanisms and implications for chronic pain. In: Graven-Nielsen T, Arendt-Nielsen L, editors. Musculoskeletal Pain: Basic Mechanisms and Implications. Washington, DC: IASP Press; 2014:471-487.

63. Cathcart S, Winefield AH, Rolan P, Lushington K. Reliability of temporal summation and diffuse noxious inhibitory control. Pain Res Manag. 2009;14(6):433-438.

64. Houben K, Jansen A. Chocolate equals stop: chocolate-specific inhibition training reduces chocolate intake and go associations with chocolate. Appetite. 2015;87:318-323. doi:10.1016/j.appet.2015.01.005

65. Gallant SN. Mindfulness meditation practice and executive functioning: breaking down the benefit. Conscious Cogn. 2016;40:116-130. doi:10.1016/j.concog.2016.01.005

66. Gomolka S, Vaegter HB, Nijs J, et al. Assessing endogenous pain inhibition: test-retest reliability of exercise-induced hypoalgesia in local and remote body parts after aerobic cycling. Pain Med. 2019;20(11):2272-2282. doi:10.1093/pm/pnz131

67. Vaegter HB, Dorge DB, Schmidt KS, Jensen AH, Graven-Nielsen T. Testretest reliability of exercise-induced hypoalgesia after aerobic exercise. Pain Med. 2018a;19(11):2212-2222. doi:10.1093/pm/pny009

68. Heathcote A, Lin Y-S, Reynolds A, Strickland L, Gretton M, Matzke D. Dynamic models of choice. Behav Res Methods. 2019;51(2):961985. doi:10.3758/s13428-018-1067-y

Journal of Pain Research

\section{Publish your work in this journal}

The Journal of Pain Research is an international, peer reviewed, open access, online journal that welcomes laboratory and clinical findings in the fields of pain research and the prevention and management of pain. Original research, reviews, symposium reports, hypothesis formation and commentaries are all considered for publication. The manuscript

management system is completely online and includes a very quick and fair peer-review system, which is all easy to use. Visit http:// www.dovepress.com/testimonials.php to read real quotes from published authors. 